\title{
Detection of explosive substances by tomographic inspection using neutron and gamma-ray spectroscopy
}

\author{
M. Farahmand, A.J. Boston, A.N. Grint, P.J. Nolan \\ M.J. Joyce, R.O. Mackin, B. D'Mellow, M. Aspinall \\ A.J. Peyton, R. van Silfhout \\ Department of Physics, University of Liverpool, UK \\ Department of Engineering, Lancaster University, UK \\ Department of Engineering, University of Manchester, UK
}

Introduction: This work discusses research and development of a technique capable of detecting and imaging hidden explosives applying Pulsed Fast Neutron Analysis (PFNA) and Compton camera techniques. We present our approach to the design and implementation of this detection system. The simulation in a Monte Carlo framework using GEANT4 has been carried out for the spectroscopy and for the imaging of gamma-ray events using the Compton camera design which is discussed. The results of Compton camera measurements using Germanium (Ge) detectors and the subsequent reconstructed images are also presented.

\section{Detection system principle}

A fast (pulsed) neutron source will be used to activate the object under investigation (Fig. 1). The prompt gamma emission from inelastic scattering will be detected, which provides a fingerprint of the explosive and thus aids of its identification. Direct imaging of the elemental contents of the material can be accomplished using the Compton camera principle. In addition detection of scattered-neutron recoils will provide a tomographic image.

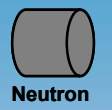
detector

dete

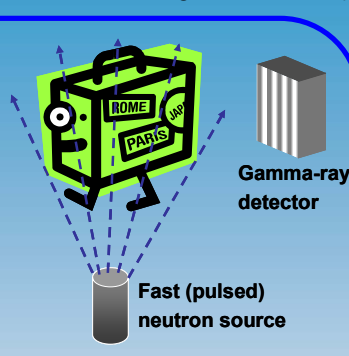

Fig. 1: A schematic view of the detection system.

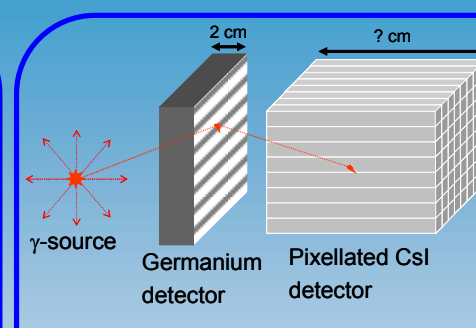

Fig. 2: A schematic view of gamma-ray detector setup. A segmented $\mathrm{Ge}$ detector will be used as scatter and a pixellated CsI detector will be applied as absorber.

\section{GEANT4 simulation results}

The GEANT4 toolkit was used for the simulation of the gamma spectroscopy and for the imaging of gammaray events using the Compton camera design. The basic setup for the simulations consists of a segmented planar Ge crystal and a pixellated Csl crystal. The former has an active volume of $60 \times 60 \times 20 \mathrm{~mm}^{3}$ which is segmented into 12 by 12 voxels of $5 \times 5 \times 20 \mathrm{~mm}^{3}$. The dimensions of the latter have been varied to study the performance of the detector. Fig. 3 displays a snapshot of the tracks of $2 \mathrm{MeV}$ gamma-rays passing through $\mathrm{Ge}$ and Csl detectors respectively, as simulated with GEANT4.

The distribution of the number of interactions per event in Ge and Csl for full energy peak events for $1 \mathrm{MeV}$ and $6 \mathrm{MeV}$ gamma-rays are shown in Fig. 4. To consider the effect of detector thickness, simulations have been carried out utilising a CsI crystal which is segmented into 8 by 8 pixels of $5.6 \times 5.6 \mathrm{~mm}^{2}$ with thicknesses of 100 and $50 \mathrm{~mm}$. The results for these two configurations are displayed in Fig. 5. Another configuration has been simulated applying Csl crystal which is segmented into 16 by 16 pixels of $5.6 \times 5.6 \times 50 \mathrm{~mm}^{3}$. A comparison of the results is shown in Fig. 6 .

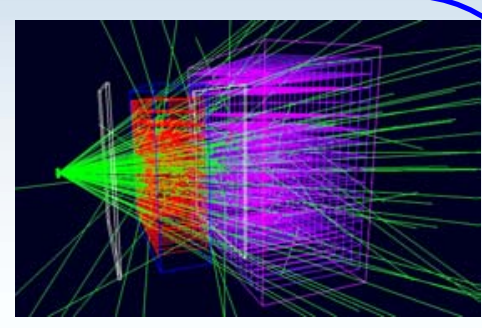

Fig. 3: A snapshot of the tracks from a gamma source of $2 \mathrm{MeV}$. The segmented planar $\mathrm{Ge}$ crystal (12 by 12) and the pixelated Csl crystal (16 by 16) are shown.
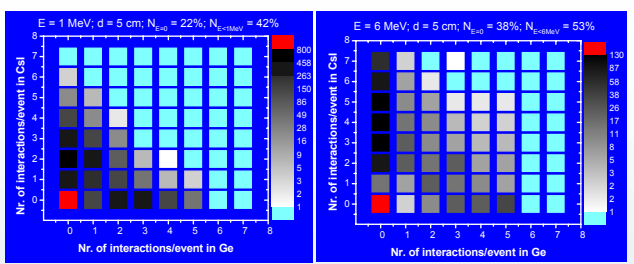

Fig. 4: Distribution of the number of interactions per event in $\mathrm{Ge}$ and $\mathrm{Cs}$ for full absorption events of $1 \mathrm{MeV}$ and $6 \mathrm{MeV}$ photons.

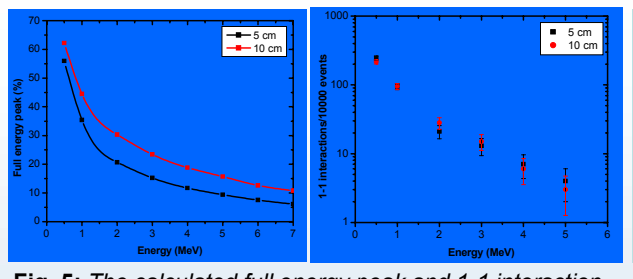

Fig. 5: The calculated full energy peak and 1-1 interaction events as a function of photon energy for pixellated Csl detector of 8 by 8 for thicknesses of $10 \mathrm{~cm}$ and $5 \mathrm{~cm}$.
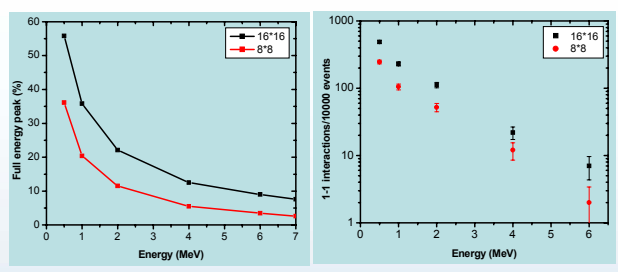

Fig. 6: Comparison of the calculated full energy peak and 1-1 interaction events as a function of photon energy for pixellated Csl detectors of 8 by 8 and 16 by 16 each having a thickness of $5 \mathrm{~cm}$.

\section{Measurement}

Compton camera measurements have been performed using two planar Ge detectors each with an active volume of $60 \times 60 \times 20$ mm ${ }^{3}$, with 12 by 12 pixel

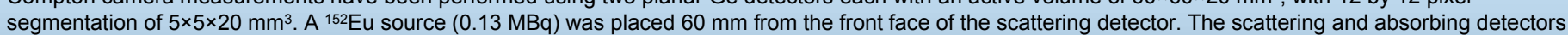
were separated by a distance of $5 \mathrm{~cm}$. The source was rotated from the normal $\left(0^{\circ}\right)$ up to $60^{\circ}$ relative to the face of the crystal in $15^{\circ}$ steps. For the $1408 \mathrm{keV}$

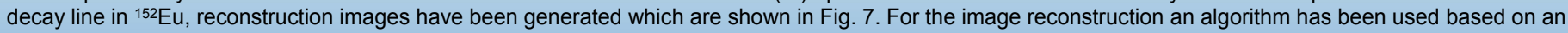
analytical technique using a back-projection method.

Fig. 7: Reconstructed images (J. Gillam, Monash University) from ${ }^{152}$ Eu point source rotating from the normal $\left(0^{\circ}\right)$ up to $60^{\circ}$ relative to the face of the detector applying two planar Ge detectors with a separation of $5 \mathrm{~cm}$.

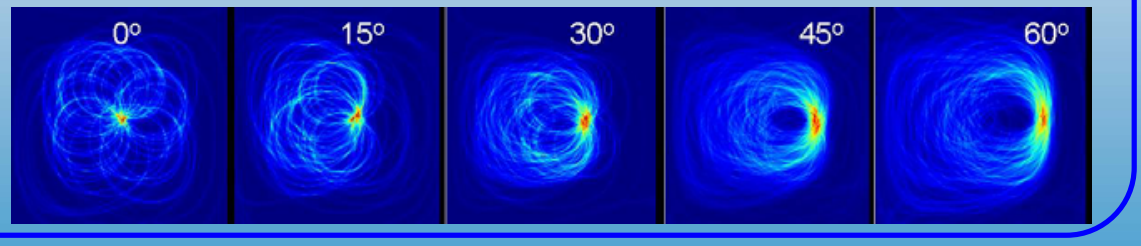

Conclusion: A technique capable of detecting and imaging hidden explosives applying PFNA and Compton camera principle has been described. The gamma-ray detector design considerations of this system which consists of a segmented Ge detector and a pixallated CsI have been discussed. The GEANT4 simulations have been carried out for various CsI detector configurations. It has been shown that reducing the depth of CsI detector from $10 \mathrm{~cm}$ to $5 \mathrm{~cm}$ decreases the detector efficiency. Utilising a physically larger CsI crystal will cause an increase in the efficiency as well as in the number of 1-1 interaction events. The Images of cone beam reconstruction applying two planar Ge detectors working in Compton camera mode have been presented.

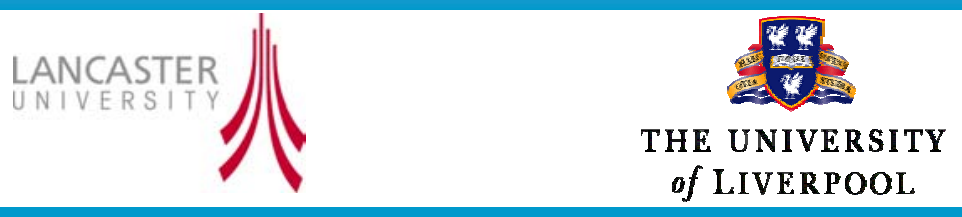

